\title{
Planet-Disk Interaction revisited
}

\author{
Manuel Jung ${ }^{1, a}$, T. F. Illenseer ${ }^{1}$, and W. J. Duschl ${ }^{1,2}$ \\ ${ }^{1}$ Institute for Theoretical Physics and Astrophysics, Christian-Albrechts-Universität Kiel, Germany \\ ${ }^{2}$ Steward Observatory, The University of Arizona, Tucson, AZ, USA
}

\begin{abstract}
We present results on our investigations of planet-disk interaction in protoplanetary disks. For the hydrodynamic simulations we use a second order semi-discrete total variation diminishing (TVD) scheme for systems of hyperbolic conservation laws on curvilinear grids.

Our previously used method conserves the momentum in two dimensional systems with rotational symmetry. Additionally, we modified our simulation techniques for inertial angular momentum conservation even in two dimensional rotating polar coordinate systems.

The basic numerical practices are outlined briefly. In addition we present the results of a common planet-disk interaction setup.
\end{abstract}

\section{2D advection solver FOSITE}

FOSITE ([1], fosite.sf.net) implements second order semi-discrete central schemes for systems of hyperbolic conservation laws on curvilinear grids. Let $(\xi, \eta, \varphi)$ be the coordinates of such a grid with the geometrical scaling factors $h_{\xi}, h_{\eta}, h_{\varphi}$. It is convenient to define new spatial differential operators

$$
\mathcal{D}_{\xi}=\frac{1}{\sqrt{g}} \frac{\partial}{\partial \xi} h_{\eta} h_{\varphi} \quad \mathcal{D}_{\eta}=\frac{1}{\sqrt{g}} \frac{\partial}{\partial \eta} h_{\xi} h_{\varphi}
$$

using the $\sqrt{g}=h_{\xi} h_{\eta} h_{\varphi}$. Systems of hyperbolic conservation laws on curvilinear grids can than be described by

$$
\partial_{t} u+\mathcal{D}_{\xi} F(u)+\mathcal{D}_{\eta} G(u)=S(u) .
$$

The numerical scheme used by FOSITE generalizes the two-dimensional central-upwind schemes developed by [2]. Geometrical source terms of vectorial conservation laws are formulated in a general prescription for various orthogonal curvilinear coordinates.

In contrast to many other astrophysical softwares used for planet-disk interaction simulations (e.g. FARGO by [3], RODEO by [4] and others in [5], as well as RAPID by [6]), FOSITE solves the Euler equations in one step without depending on techniques such as operator splitting.

In conclusion we abstract the most import features of FOSITE:

- finite volume scheme for hyperbolic conservation laws

\footnotetext{
a e-mail: mjung@astrophysik.uni-kiel.de
} 
- semi discrete: second order in space, up to fifth order in time

- total variation diminishing with a variety of flux limiters

- arbitrary orthogonal curvilinear grids

- upwind: accounts for propagation of information

- Fortran 95, object-oriented design, GPL

- parallelised using MPI, vectorized for NEC SX8/9

- outputs: ASCII, gnuplot, VTK, netcdf, hdf5, binary

- integrated python based plotting framework

\section{Inertial angular momentum transport}

If exact conservation of angular momentum in the inertial frame is of great interest, it is possible to reformulate the Euler equations for transport of inertial angular momentum $l$. This includes the Coriolis and centrifugal forces in the rotating frame of reference with angular velocity $\Omega$ :

$$
l=h_{\eta}\left(v_{\eta}+h_{\eta} \Omega\right)
$$

Here $h_{\eta}$ is the geometrical scaling factor along the second coordinate, e.g. for polar coordinates $h_{\eta}=r$. This leads to a new system of hyperbolic conservation laws, which imply exact conservation of mass and inertial angular momentum. Using the local isothermal speed of sound approximation yields $p=\rho c_{s}^{2}$ for the pressure, we derive:

$$
\begin{gathered}
u=\left[\begin{array}{c}
\rho \\
\rho v_{\xi} \\
\rho l
\end{array}\right], \quad F=\left[\begin{array}{c}
\rho v_{\xi} \\
\rho v_{\xi}^{2}+p \\
\rho v_{\xi} l
\end{array}\right], \quad G=\left[\begin{array}{c}
\rho v_{\eta} \\
\rho v_{\xi} v_{\eta} \\
\rho v_{\eta} l+h_{\eta} p
\end{array}\right], \\
S=\left[\begin{array}{c}
\rho h_{\eta}\left(\frac{v_{\eta}}{h_{\eta}}+\Omega\right)^{2}+p \frac{1}{h_{\eta}} \frac{\partial h_{\eta}}{\partial \xi} \\
0
\end{array}\right] .
\end{gathered}
$$

\section{Planet-disk interactions}

We validate FOSITE with the planet-disk interaction standard simulations as proposed by [5]. First we compare a standard jupiter mass simulation using inertial angular momentum transport to our past method. The figures shown below display the surface densities and the radial density profiles after 30 and 100 planet orbits. While the depth of the gap is similar in both simulations, there is a big difference at the gap edges in the radial density plot. Furthermore the Lagrange points L4 and L5 can only be seen in the simulation with inertial angular momentum transport, since the other is a lot more diffusive.

Secondly we compare results using the standard resolution $128 \times 384$ to high resolution $512 \times 1536$ simulations. Generally these simulations agree quite well. However the high resolution simulation shows different behavior regarding the Lagrange points, which vanish a lot faster in the standard simulations. In the high resolution simulation the outer edge of the gap is dominated by a big vortex which rotates with a lower angular velocity than the proto planet. 

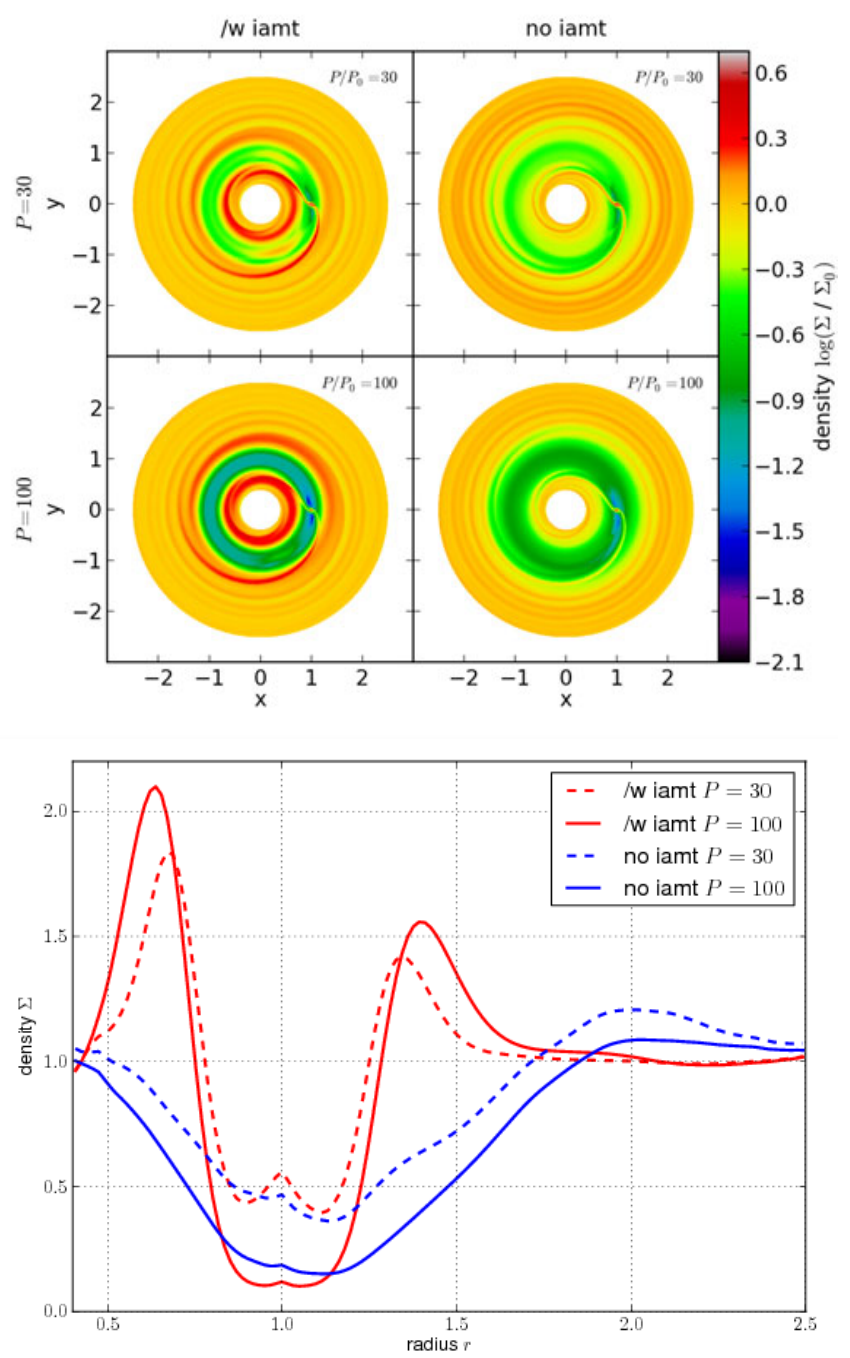

Figure 1. Comparison of planet-disk interaction simulations with and without inertial angular momentum transport (iamt) after 30 and 100 planetary orbits.

\section{Conclusions}

We could show, that exact inertial angular momentum conservation can be achieved without relying on techniques like operator splitting. FOSITEs results agree well with standard simulations. In the high resolution runs we observe a big vortex at the outer gap edge. 

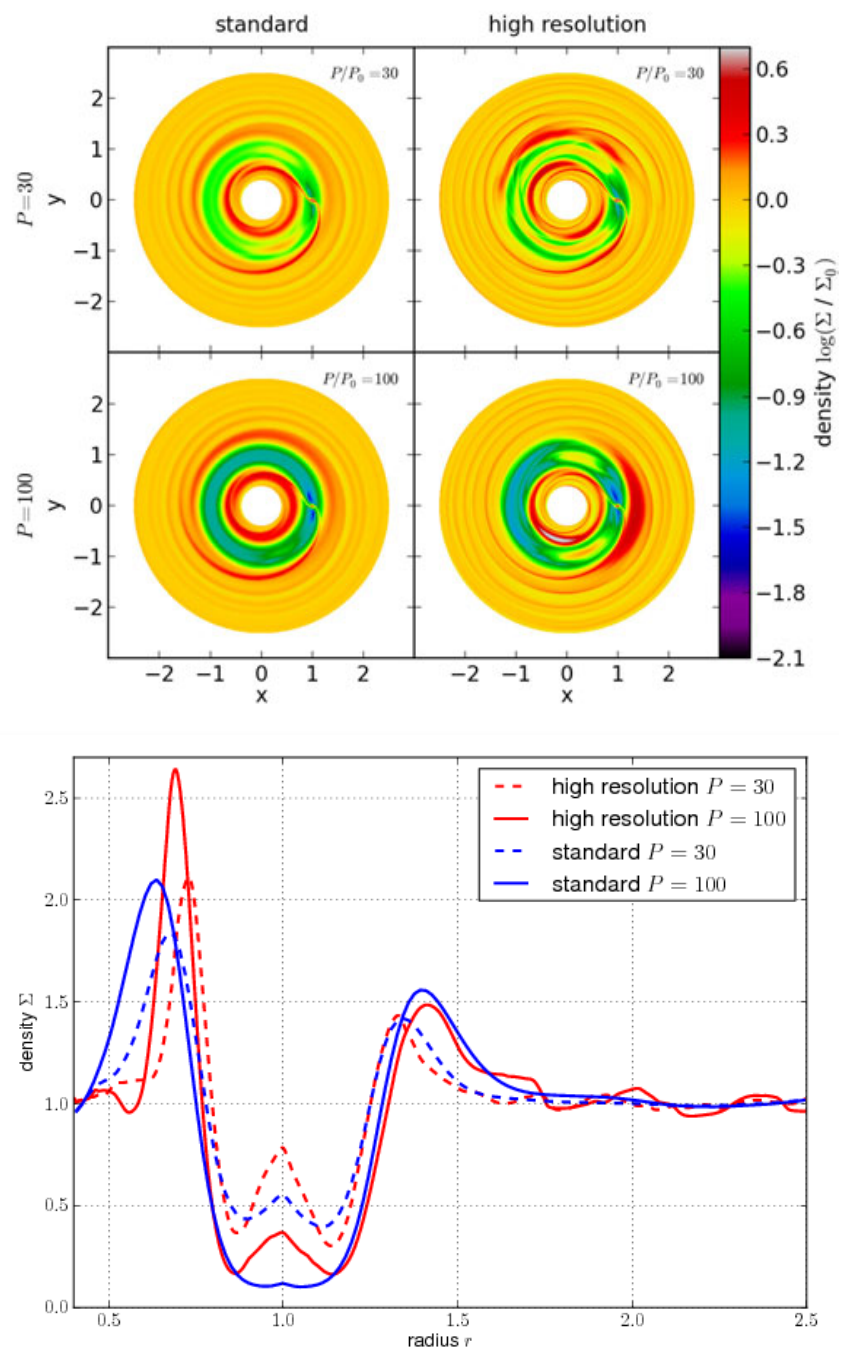

Figure 2. Comparison of planet-disk interaction simulations with standard and high resolution after 30 and 100 planetary orbits.

\section{References}

[1] T.F. Illenseer, W.J. Duschl, CPC 180, 2283 (2009), 0804 . 2979

[2] A. Kurganov, E. Tadmor, JCP 160, 241 (2000)

[3] F. Masset, AAPS 141, 165 (2000), arXiv: astro-ph/9910390

[4] S.J. Paardekooper, G. Mellema, A\&A 450, 1203 (2006), arXiv:astro-ph/0511538

[5] M. de Val-Borro et al., MNRAS 370, 529 (2006), arXiv: astro-ph/0605237

[6] L.R. Mudryk, N.W. Murray, NA 14, 71 (2009), 0812 . 2938 